УДК 621.396 .96

\title{
АДАПТИВНОЕ ПОСЛЕДОВАТЕЛЬНОЕ ОБНАРУЖЕНИЕ ТРАЕКТОРИИ ЦЕЛИ С ИСПОЛЬЗОВАНИЕМ РЕШАЮЩИХ СТАТИСТИК ОТМЕТОК ПРИ НЕИЗВЕСТНОМ ОТНОШЕНИИ СИГНАЛ-ШУМ
}

\author{
НЕУЙМИН А. С., ЖУК С. Я. \\ Национальный технический университет Украинь \\ «Киевский политехнический институт», \\ Украина, Киев, 03056, пр-т Победь 37
}

\begin{abstract}
Аннотация. На основе последовательного критерия простого дополнения получены адаптивные алгоритмы многоальтернативного последовательного обнаружения траектории цели по верхним и нижним порогам с использованием решающих статистик отметок при неизвестном отношении сигнал-шум (ОСШ). Применение нижних порогов позволило реализовать процедуру отбрасывания неудачных гипотез. Разработан адаптивный алгоритм двухальтернативного последовательного обнаружения траектории цели с использованием решающих статистик отметок с оцениванием ОСШ по критерию минимума среднего квадрата ошибки. Анализ алгоритмов выполнен с помощью статистического моделирования на примере обнаружения траектории цели по данным обзорной РЛС, измеряющей дальность и радиальную скорость цели.
\end{abstract}

Ключевые слова: обнаружение траектории цели; неизвестное отношение сигнал-шум; критерий Вальда; решающие статистики отметок; функция правдоподобия

\section{ВВЕДЕНИЕ}

Широкое распространение для оптимального обнаружения траектории цели находит последовательный критерий Вальда [1], который позволяет получить существенный выигрыш во времени при заданных требованиях к вероятностям принимаемых решений. В [2] на его основе получены алгоритмы последовательного обнаружения траектории цели с использованием решающих статистик отметок в стробе сопровождения при известном отношении сигнал-шум (ОСШ).

Критерий Вальда теряет свои оптимальные свойства если параметр, характеризующий фактическое состояние исследуемого процесса, принимает промежуточное значение между значениями, заданными в проверяемых гипотезах [3]. Поэтому, если фактическое
ОСШ цели ниже заданного, то эффективность обнаружения траектории цели резко снижается.

Необходимо отметить, что в разработанных методах сопровождения цели при наличии ложных отметок с использованием амплитудной информации [4] также полагается, что ОСШ является известным. Несоответствие фактического ОСШ заданному может приводить к увеличению вероятности сброса траектории, обнаруженной «слабой» цели, а также к росту времени обнаружения срыва сопровождения цели. Кроме того, оценивание ОСШ цели также имеет важное значение для ряда приложений, поскольку может быть использовано для распознавания ее класса.

Поэтому важное значение имеет разработка адаптивных алгоритмов обнаружения тра- 